Saharon Shelah, Institute of Mathematics, Hebrew University

\title{
COVERING NUMBERS ASSOCIATED WITH TREES BRANCHING INTO A COUNTABLY GENERATED SET OF POSSIBILITIES
}

\begin{abstract}
One can think of the dominating number as the covering number for the ideal on the $\omega$-branching tree generated by finite branching subtrees. This paper is concerned with generalizations of this when "finite" is replaced by some other concept. A key example is obtained by thinking of the branching as being into the integers — both positive and negative - and replacing "finite" by "bounded either above or below". This notion was motivated by considerations related to decomposing functions of low Baire class into continuous functions.
\end{abstract}

\section{Introduction}

This paper is concerned with certain generalizations of meagerness and their combinatorial equivalents. The simplest example, and the one which motivated further study in this area, comes about by considering the following definition.

Definition 1. For any $A \subseteq \mathbb{R}$ a set $X \subseteq \mathbb{R}$ will be said to be $A$-nowhere dense $^{1}$ if and only if for every $q \in A$ there exists and integer $k \in \mathbb{Z}$ such that the interval whose endpoints are $q$ and $q+1 / k$ is disjoint from $X$. A set which is the union of countably many $A$-nowhere dense sets will be called $A$-very meager.

\footnotetext{
Mathematical Reviews subject classification: Primary 03E35; Secondary 28A05

Received by the editors June 11, 1997

* The author would like to thank Juris Steprāns for writing up this paper. This paper is number 660 in the author's personal listing

${ }^{1}$ This notation corrects the terminology of [4] which called a special case of this notion almost nowhere dense in spite of the fact that almost nowhere dense sets are nowhere dense rather than the converse.
} 
The notion of an $A$-nowhere dense set for various subsets $A$ of the reals may prove to be of interest in its own right, but this paper will be concerned exclusively with the special case $A=\mathbb{Q}$. Note that rational perfect sets, introduced by Miller in [1], form a subset of the $\mathbb{Q}$-nowhere dense sets since the closure of a set is rational perfect if it is perfect and disjoint from the rationals. On the other hand, a set is $\mathbb{Q}$-nowhere dense if its 2 -sided-closure is disjoint from the rationals where the 2-sided-closure of a set refers to all those reals which are limits of both decreasing and increasing sequences from the set.

In [4] the least number of $\mathbb{Q}$-meager sets required to cover the real line is examined and is denoted by $\mathfrak{d}_{1}$. It is shown in [3] that there is a continuous function $H$ - first constructed by Lebesgue - such that the least number of smooth functions into which $H$ can be decomposed is equal to the $\mathfrak{d}_{1}$. This paper will further study $\mathfrak{d}_{1}$ and some of its generalizations. As well, an equivalence will be established between $\mathbb{Q}$-meagerness and certain combinatorial properties of trees. This will lead to new cardinal invariants and various independence results about these will then be established.

\section{Equivalences}

Definition 2. A set $X \subseteq \omega \mathbb{Z}$ is small if for each $n \in \omega$ and $\sigma: n \rightarrow \mathbb{Z}$ there is some $k \in \mathbb{Z}$ such that either

$$
\{f(n): f \in X \text { and } \sigma \subseteq f\} \subseteq\{m \in \mathbb{Z}: m<k\}
$$

or

$$
\{f(n): f \in X \text { and } \sigma \subseteq f\} \subseteq\{m \in \mathbb{Z}: m>k\} .
$$

It will be shown that the least number of very meager sets required to cover $\mathbb{R}$ is equal to the least number of small sets required to cover $\omega \mathbb{Z}$. This is an immediate consequence of the following lemma. The idea is to modify one of the standard ${ }^{2}$ constructions of a homeomorphism between $\omega_{\omega}$ and $\mathbb{R} \backslash \mathbb{Q}$. In this construction $\omega_{\omega}$ is identified with $\omega_{\mathbb{Z}}$ and finite sequences $\sigma: m \rightarrow \mathbb{Z}$ are mapped to open intervals $I(\sigma)$ so that

- if $\sigma \subseteq \tau$, then $I(\sigma) \supseteq I(\tau)$

- $\bigcup_{n \in \mathbb{Z}} \overline{I\left(\sigma^{\wedge} n\right)}=I(\sigma)$

- the left endpoint of $I\left(\sigma^{\wedge} n\right)$ is the right endpoint of $I\left(\sigma^{\wedge}(n-1)\right)$.

${ }^{2}$ For example see page 5 of [2]. 
This construction will be modified by, essentially, mapping sequences which end with a negative integer to their counterpart above the neighbor to the right of their parent sequence. The details are explained in the following.

Lemma 1. Let $X \subseteq{ }^{\mathbb{Z}}$ be the set of all sequences eventually equal to -1 together with the constant sequence 0 . There is a bijection $F:{ }^{\mathbb{Z}} \backslash X \rightarrow \mathbb{R} \backslash \mathbb{Q}$ such that $A \subseteq \mathbb{R} \backslash \mathbb{Q}$ is very nowhere dense if and only if $F^{-1} A$ is small.

Proof. To begin, let $\left\{q_{n}\right\}_{n \in \omega}$ enumerate $\mathbb{Q}$ so that each rational occurs infinitely often. Let $\stackrel{\omega}{\omega}^{-} \mathbb{Z}$ denote the set of finite sequences of integers - in other words, the set of functions from an integer to $\mathbb{Z}$. For $\sigma \in \stackrel{\omega}{\omega}^{\mathbb{Z}}$ define $\mu(\sigma)=\sigma(n)$ where $n$ is the greatest integer in the domain of $\sigma$. Let $\prec$ be the partial ordering of $\stackrel{\omega}{*}_{\mathbb{Z}}$ defined by $\sigma \prec \tau$ if

- there exists some least integer $n$ such that $\sigma(n) \neq \tau(n)$ and $0 \leq \tau(n)<$ $\sigma(n)$

- there exists some least integer $n$ such that $\sigma(n) \neq \tau(n)$ and $\tau(n)<$ $\sigma(n)<0$

- there exists some least integer $n$ such that $\sigma(n) \neq \tau(n)$ and $\sigma(n)<0 \leq$ $\tau(n)$.

For $\sigma \in{ }^{{ }^{\omega}} \mathbb{Z}$ and $n \in \mathbb{Z}$ define $\sigma^{n} \in{ }^{\omega} \mathbb{Z}$ such that $\sigma$ and $\sigma^{n}$ have the same domain, $\sigma(i)=\sigma^{n}(i)$ if $i$ is not the maximal element of the common domain of $\sigma$ and $\sigma^{n}$ and $\mu(\sigma)+n=\mu\left(\sigma^{n}\right)$. Moreover, let $\sigma^{-}=\sigma \uparrow(|\sigma|-1)$. Next,

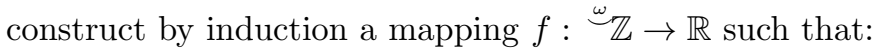

- $f$ is a $\prec$ order preserving mapping whose range is disjoint from $\mathbb{Q}$

- if $\mu(\sigma) \neq-1$ and $\sigma^{-}$is -1 on its domain, or $\sigma^{-} \not \equiv 0, \sigma^{-} \not \equiv-1, \mu(\sigma) \geq 0$, then $f\left(\sigma^{1}\right)<f\left(\sigma^{\wedge} i\right)<f(\sigma)$ for each $i \in \mathbb{Z} \backslash\{0,-1\}$ and $f\left(\sigma^{\wedge}-1\right)=$ $f\left(\sigma^{1}\right), f\left(\sigma^{\wedge} 0\right)=f(\sigma)$.

- if $\mu(\sigma) \neq 0$ and $\sigma^{-}$is 0 on its domain or $\sigma^{-} \not \equiv 0, \sigma^{-} \not \equiv-1 \mu(\sigma)<0$, then $f(\sigma)<f\left(\sigma^{\wedge} i\right)<f\left(\sigma^{-1}\right)$ for each $i \in \mathbb{Z} \backslash\{0,-1\}$, and $f\left(\sigma^{\wedge}-1\right)=$ $f(\sigma), f\left(\sigma^{\wedge} 0\right)=f\left(\sigma^{-1}\right)$.

- $\lim _{n \in \omega} f\left(\sigma^{n}\right)=\lim _{n \in \omega} f\left(\sigma^{-n}\right) \in \mathbb{Q}$ for every $\sigma \in{ }^{\omega}{ }^{\omega} \mathbb{Z}$

- $|f(\sigma)-f(\tau)|<\frac{1}{k+1}$ for every $\sigma$ and $\tau$ in ${ }^{k} \mathbb{Z}$ such that $\tau$ is the immediate successor of $\sigma$ with respect to $\prec$ in ${ }^{k} \mathbb{Z}$

- if $\sigma \equiv 0$, then $f\left(\sigma^{\wedge} 0\right)=f(\sigma)+\frac{1}{|\sigma|+1}, f\left(\sigma^{\wedge}-1\right)=f(\sigma)$ 
- if $\sigma \equiv-1$, then $f\left(\sigma^{\wedge}-1\right)=f(\sigma)-\frac{1}{|\sigma|+1}, f\left(\sigma^{\wedge} 0\right)=f(\sigma)$

- if $\sigma, \tau \in k_{\mathbb{Z}}$ are two successive sequences (with respect to $\prec$ ), and both $q_{k}$ and $f\left(\sigma^{\wedge} 1\right)$ are between $f(\sigma)$ and $f(\tau)$, then $\lim _{n \in \omega} f\left(\sigma^{\wedge} n\right)=$ $\lim _{n \in \omega} f\left(\sigma^{\wedge}-n\right)=q_{k}$.

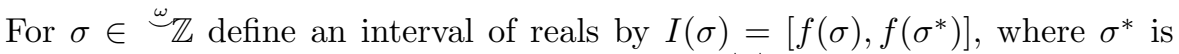
suitably either successor or predecessor of $\sigma$ in $|\sigma| \mathbb{Z}$ and define $F:{ }^{\omega} \backslash X \rightarrow$ $\mathbb{R} \backslash \mathbb{Q}$ by taking the intersection along a branch - in other words, $F(\sigma)$ is the unique element of $\bigcap_{n \in \omega} I(\sigma\lceil n)$. It is easy to check that this mapping has the desired properties.

\section{Trees of Countable Structures}

A cover on a countable set $X$ is a countable subset $\mathcal{B} \subseteq \mathcal{P}(X)$ such that

- $X \notin \mathcal{B}$

- if $B \in \mathcal{B}$ and $b \in[X]^{<\aleph_{0}}$, then $B \cup b \in \mathcal{B}$.

For a cover $\mathcal{B}$ on $X$ define $\overline{\mathcal{B}}=\{Y \subseteq X:(\exists A \in \mathcal{B})(Y \subseteq A)\}$ and define $\mathcal{B}^{+}=\mathcal{P}(X) \backslash \overline{\mathcal{B}}$. Define a $\mathcal{B}$-tree to be a tree $T \subseteq{ }^{\omega} X$ such that for each $t \in T$ the set of successors of $t$ in $T$ belongs to $\overline{\mathcal{B}}$ or, to be more precise, $\{s(|t|): s \in T$ and $t \subsetneq s\} \in \overline{\mathcal{B}}$. The notation ${ }^{\omega} \omega$ will be used to denote the set of all functions from a proper, initial segment of $\omega$ to $\omega$. Finally, define $\mathcal{J}_{\mathcal{B}}$ to be the $\sigma$-ideal generated by all sets $X \subseteq{ }^{\omega}{ }^{\omega} \omega$ such that there is some $\mathcal{B}$-tree $T$ such that $X \subseteq \bar{T}=\{f:(\forall n \in \omega) f\lceil n \in T\}$.

The examples of covers with which this paper will be concerned are of the form

$$
\mathcal{B}_{n}=\left\{A \subseteq \omega \times n:(\exists i \in n)|\{m \in \omega:(m, i) \in A\}|<\aleph_{0}\right\}
$$

although many other examples are possible. It will be shown that for any integer $m>1$ it is consistent that $\operatorname{cov}\left(\mathcal{J}_{\mathcal{B}_{m}}\right)=\omega_{2}$ but $\operatorname{cov}\left(\mathcal{J}_{\mathcal{B}_{m+1}}\right)=\omega_{1}$.

Definition 3. For a cover $\mathcal{B}$ on a set $X$ define $\mathbb{P}(\mathcal{B})$ to be the set of all triples $(t, F, \Gamma)$ such that:

1. $t \subseteq{ }^{\omega} X$ is a finite subtree - in particular, $t$ is closed under initial segments

2. $F: t \rightarrow \mathcal{B}$

3. $\Gamma \in\left[{ }^{\omega} X\right]<\aleph_{0}$ 
4. there is a one-to-one function $\beta: \Gamma \rightarrow t$ which maps $\Gamma$ onto the maximal nodes of $t$ such that $\beta(x) \subseteq x$ for all $x \in \Gamma$

5. $x(n) \in F(x\lceil n)$ for every $x \in \Gamma$ and for every $n \in \omega$ such that $x\lceil n \in t$.

The ordering on $\mathbb{P}(\mathcal{B})$ is coordinatewise containment. Observe that if $(t, F, \Gamma)$ and $\left(t^{\prime}, F^{\prime}, \Gamma^{\prime}\right)$ are in $\mathbb{P}(\mathcal{B})$ and $t=t^{\prime}, F=F^{\prime}$, then the two conditions are compatible.

If $\mathcal{B}$ is a cover on $X$ and $\mathcal{C}$ is a cover on $Y$, then define $\mathcal{B} \prec \mathcal{C}$ if and only if for every $A \in \mathcal{B}^{+}$and for every $H: A \rightarrow{ }^{\omega} Y$ there is $B \subseteq A$ such that $B \in \mathcal{B}^{+}$ such that there is a finite $t \subseteq{ }^{\omega} Y$ and a mapping $F: t \rightarrow \mathcal{C}$ and there is a finite set $C \subseteq{ }^{\omega} Y$ and $B_{0} \cup B_{1} \subseteq B$ such that $B_{0} \cup B_{1} \in \mathcal{B}^{+}$and

$$
\begin{gathered}
\left(\forall\left\{b, b^{\prime}\right\} \in\left[B_{0}\right]^{2}\right)(\exists \tau \in t)\left(\exists\left\{y, y^{\prime}\right\} \in[F(\tau)]^{2}\right)\left(\tau^{\wedge} y \subseteq H(b) \text { and } \tau^{\wedge} y^{\prime} \subseteq H\left(b^{\prime}\right)\right) \\
(\forall n \in \omega)\left(\mid\left\{b \in B_{1}:(\forall c \in C)(H(b)\lceil n \not \subset c)\} \mid<\aleph_{0}\right)\right.
\end{gathered}
$$

Definition 4. A $(\mathcal{J}, \kappa)$-Lusin set for an ideal $\mathcal{J}$ on a set $X$ is a set $L \subseteq X$ such that $|L \cap J|<\kappa$ for all $J \in \mathcal{J}$.

Lemma 2. If $\mathcal{B}$ and $\mathcal{C}$ are covers on $X$ and $Y$ respectively, $\mathcal{B} \prec \mathcal{C}, \kappa$ is a cardinal of uncountable cofinality and $W$ is a $\left(\mathcal{J}_{\mathcal{B}}, \kappa\right)$-Lusin set, then $1 \Vdash_{\mathbb{P}(\mathcal{C})}$ "W is a $\left(\mathcal{J}_{\mathcal{B}}, \kappa\right)$-Lusin set".

Proof. If not, then let $T$ be a $\mathbb{P}(\mathcal{C})$-name for $\mathcal{B}$-tree such that

$$
1 \Vdash_{\mathbb{P}(\mathcal{C})} "\left\{w_{\alpha}\right\}_{\alpha \in \kappa} \subseteq \bar{T} \cap W \text { and } w_{\alpha} \neq w_{\beta} \text { if } \alpha \neq \beta "
$$

and, for each $\alpha \in \kappa$, choose $\left(t_{\alpha}, F_{\alpha}, \Gamma_{\alpha}\right) \in \mathbb{P}(\mathcal{C})$ deciding the value of $w_{\alpha}-$ in other words, such that there is $y_{\alpha}$ such that $\left(t_{\alpha}, F_{\alpha}, \Gamma_{\alpha}\right) \Vdash_{\mathbb{P}(\mathcal{C})}$ " $w_{\alpha}=\check{y}_{\alpha}$ ". Then, using the fact that $\kappa$ has uncountable cofinality, choose $t$ and $F$ such that $W^{*}=\left\{y_{\alpha}: t=t_{\alpha}\right.$ and $\left.F=F_{\alpha}\right\}$ has cardinality $\kappa$. It follows $W^{*}$ is also a $\left(\mathcal{J}_{\mathcal{B}}, \kappa\right)$-Lusin set and, hence, that it is in $\mathcal{J}_{\mathcal{B}}^{+}$.

Next, choose $m \in \omega$ and $\sigma: m \rightarrow \omega$ such that

$$
S_{0}=\left\{s \in X:\left(\exists w \in W^{*}\right)\left(\sigma^{\wedge} s \subseteq w\right)\right\} \in \mathcal{B}^{+}
$$

and choose $W^{\prime} \subseteq W^{*}$ such that for each $s \in S_{0}$ there is a unique $w_{s} \in W^{\prime}$ such that $w_{s}(m)=s$. Let $\alpha(s)$ be the unique ordinal such that $w_{s}=y_{\alpha(s)}$. Let the maximal nodes of $t$ be enumerated by $\left\{\nu_{i}\right\}_{i=1}^{k}$ and let $b_{i}^{s}$ be the unique member of $\Gamma_{\alpha(s)}$ such that $\nu_{i} \subseteq b_{i}^{s}$. Proceed by induction to define $S_{0}^{i}$ and $S_{1}^{i}$ for $i \leq k$ so that if $S_{0}^{i}$ and $S_{1}^{i}$ have been chosen, then $S_{0}^{i+1} \cup S_{1}^{i+1} \subseteq S_{i}=S_{0}^{i} \cup S_{1}^{i}$ is 
chosen so that $S_{0}^{i+1} \cup S_{1}^{i+1} \in \mathcal{B}^{+}$and so that there is a finite $r_{i+1} \subseteq{ }^{\omega} Y$ and a mapping $F_{i+1}: r_{i+1} \rightarrow \mathcal{C}$ and there is a finite set $L_{i+1} \subseteq{ }^{\omega} Y$ such that

$$
\begin{gathered}
\left(\forall\{s, \bar{s}\} \in\left[S_{0}^{i+1}\right]^{2}\right)\left(\exists \tau \in r_{i+1}\right)\left(\exists\{y, \bar{y}\} \in\left[F_{i+1}(\tau)\right]^{2}\right)\left(\tau^{\wedge} y \subseteq b_{i+1}^{s}\right. \\
\text { and } \left.\tau^{\wedge} \bar{y} \subseteq b_{i+1}^{\bar{s}}\right) \\
(\forall n \in \omega)\left(\mid\left\{s \in S_{1}^{i+1}:\left(\forall c \in L_{i+1}\right)\left(b_{i+1}^{s}\lceil n \not c)\right\} \mid<\aleph_{0}\right)\right. \\
\nu_{i} \subseteq \bigcap\left(r_{i} \cup L_{i}\right) \\
F_{i}\left(\nu_{i}\right)=F\left(\nu_{i}\right) \\
\left(\forall c \in L_{i}\right)\left(\exists \sigma \in \max \left(r_{i}\right)\right)(\sigma \subseteq c) \\
\left(\forall c \in L_{i}\right)\left(\forall \sigma \in r_{i}\right)(\sigma \subseteq c \rightarrow c(|\sigma|) \in F(\sigma)) \\
\text { if } \tau \in r_{i+1} \text { and } n<|\tau|, \text { then } \tau(n) \in F_{i+1}(\tau\lceil n) .
\end{gathered}
$$

This is easily accomplished using the definition of $\mathcal{B} \prec \mathcal{C}$. Then define a new condition $\left(t^{\prime}, F^{\prime}, \Gamma^{\prime}\right)$ such that $t \cup \bigcup_{i=1}^{k} r_{i} \subseteq t^{\prime}, F \cup \bigcup_{i=1}^{k} F_{i} \subseteq F^{\prime}$ and $\Gamma \cup \bigcup_{i=1}^{k} L_{i} \subseteq \Gamma^{\prime}$.

It suffices to show that $\left(t^{\prime}, F^{\prime}, \Gamma^{\prime}\right) \Vdash_{\mathbb{P}(\mathcal{C})}$ " $\left\{s \in S_{k}: w_{s} \in \bar{T}\right\} \notin \overline{\mathcal{B}}$ " because this would contradict that $1 \Vdash_{\mathbb{P}(\mathcal{C})}$ " $T$ is a $\mathcal{B}$-tree". Therefore suppose that $\left(t^{\prime \prime}, F^{\prime \prime}, \Gamma^{\prime \prime}\right)$ is a condition extending $\left(t^{\prime}, F^{\prime}, \Gamma^{\prime}\right)$ such that

$$
\left(t^{\prime \prime}, F^{\prime \prime}, \Gamma^{\prime \prime}\right) \Vdash_{\mathbb{P}(\mathcal{C})} \text { " }\left\{s \in S_{k}: w_{s} \in \bar{T}\right\} \subseteq B "
$$

for some $B \in \mathcal{B}$. In order to obtain a contradiction, notice that for each $i$ the set $\Lambda_{0}^{i}=\left\{s \in S_{k} \cap S_{0}^{i}:\left(\exists \tau \in t^{\prime \prime}\right)\left(\tau \subseteq b_{i}^{s}\right.\right.$ and $\left.\left.b_{i}^{s}(|\tau|) \notin F_{i}^{\prime \prime}(\tau)\right)\right\}$ is finite because of (3.1) and the fact that $r_{i} \subseteq t^{\prime \prime}$. On the other hand, it is possible to choose $N$ so large that for each $i$ and each $c \in L_{i}$ the sequence $c\lceil N$ does not belong to $t^{\prime \prime}$. Then for each $i$ the set

$$
\Lambda_{1}^{i}=\left\{s \in S_{k} \cap S_{1}^{i}:\left(\forall c \in L_{i}\right)\left(b_{i}^{s} \uparrow N \nsubseteq c\right)\right\}
$$

is also finite. Therefore it is possible to choose $s \in S_{k} \backslash\left(B \cup \bigcup_{i=1}^{k}\left(\Lambda_{0}^{i} \cup \Lambda_{1}^{i}\right)\right)$ and let $\Gamma^{*}=\Gamma^{\prime \prime} \cup\left\{b_{i}^{s}\right\}_{i=1}^{k}$.

First note that it is easy to extend $t^{\prime \prime}$ to $t^{*}$ and $F^{\prime \prime}$ to $F^{*}$ so that $\left(t^{*}, F^{*}, \Gamma^{*}\right)$ satisfies Conditions 1, 2 and 4 of Definition 3 because $[Y]^{<\aleph_{0}} \subseteq \mathcal{C}$. Since Condition 3 is also satisfied it suffices to show that $\left(t^{*}, F^{*}, \Gamma^{*}\right)$ satisfies Condition 5 in Definition 3. This, in turn, follows from consideration of the two cases. First, if $s \in S_{0}^{i}$, then $s \notin \Lambda_{0}^{i}$ and so there is some $\tau \in r_{i}$ and $y \in F_{i}(\tau)=F^{\prime \prime}(\tau)=F^{*}(\tau)$ such that $\tau^{\wedge} y \subseteq b_{i}^{s}$ and $\tau^{\wedge} y \notin t^{\prime \prime}$. Hence there is no contradiction to Condition 5 because any such contradiction would already have occurred in the 
condition $\left(t_{\alpha(s)}, F_{\alpha(s)}, \Gamma_{\alpha(s)}\right)$. If $s \in S_{1}^{i}$, then there is some $c \in L_{i} \subseteq \Gamma^{\prime \prime}$ such that $b_{i}^{s} \uparrow N=c\lceil N$ and $c$ does not violate Condition 5. Moreover the choice of $N$ guarantees that $c$ violates Condition 5 if and only if $b_{i}^{s}$ does. But now $\left(t^{*}, F^{*}, \Gamma^{*}\right)$ obviously extends $\left(t, F, \Gamma_{\alpha(s)}\right)=\left(t_{\alpha(s)}, F_{\alpha(s)}, \Gamma_{\alpha(s)}\right)$ which forces that $w_{s} \in \bar{T}$ and, hence, that $s \in B$ and this is a contradiction.

Corollary 1. Suppose that $\mathcal{B}$ is a cover and that $W$ is a $\left(\mathcal{J}_{\mathcal{B}}, \kappa\right)$-Lusin set. Suppose also that $\lambda \in \kappa$ and $\operatorname{cof}(\kappa)>\aleph_{0}$ and that $\left\{\mathcal{C}_{\alpha}\right\}_{\alpha \in \lambda}$ are covers such that $\mathcal{B} \prec \mathcal{C}_{\alpha}$ for each $\alpha \in \lambda$. If, furthermore, $\mathbb{P}$ is the finite support iteration of $\left\{\mathbb{P}\left(\mathcal{C}_{\alpha}\right)\right\}_{\alpha \in \lambda}$, then $1 \Vdash_{\mathbb{P}}$ "W is a $\left(\mathcal{J}_{\mathcal{B}}, \kappa\right)$-Lusin set".

Proof. Proceed by induction on $\lambda$. If $\lambda$ is a limit ordinal and the lemma fails, then let $G \subset \mathbb{P}$ be generic and choose a $\mathcal{J}_{\mathcal{B}}$-tree $T$ and $\left\{p_{\alpha}\right\}_{\alpha \in \kappa} \subseteq G$ such that $p_{\alpha} \Vdash_{\mathbb{P}}$ " $\hat{w}_{\alpha} \in \bar{T} \cap W$ " and such that the $w_{\alpha}$ are all distinct. Then use the finite support of the iteration and the fact that the cofinality of $\lambda$ is less than $\kappa$ to conclude that there is some $\beta \in \alpha$ such that $G \cap \mathbb{P}_{\beta}$ - where $\mathbb{P}_{\beta}$ is the finite support iteration of $\left\{\mathbb{P}\left(\mathcal{C}_{\nu}\right)\right\}_{\nu \in \beta}$ - contains $\kappa$ of the conditions $\left\{p_{\alpha}\right\}_{\alpha \in \kappa}$. This contradicts the induction hypothesis because the closure of the corresponding $w_{\alpha}$ will form a $\mathcal{J}_{\mathcal{B}}$-tree contained in $\bar{T}$.

At successors, use the induction hypothesis, Lemma 2 and the absoluteness of the relation $\prec$.

Lemma 3. If $\mathbb{P}$ is the finite support iteration of length $\omega$ of the partial orders $\mathbb{P}(\mathcal{B})$ for some cover $\mathcal{B}$ and $G$ is $\mathbb{P}$ generic over $V$, then, in $V[G]$, there are countably many sets in $\mathcal{J}_{\mathcal{B}}$ whose union covers $V \cap{ }^{\omega}{ }_{\omega}$.

Proof. Standard.

Theorem 1. If $\kappa_{0}>\kappa_{1}$ are uncountable regular cardinals and $\mathcal{B}_{0}$ and $\mathcal{B}_{1}$ are covers such that $\mathcal{B}_{0} \prec \mathcal{B}_{1}$, then it is consistent that $\operatorname{cov}\left(\mathcal{J}_{\mathcal{B}_{0}}\right)=\kappa_{0}$ and $\operatorname{cov}\left(\mathcal{J}_{\mathcal{B}_{1}}\right)=\kappa_{1}$

Proof. Let $V \models$ GCH \& ZFC and let $\mathbb{P}_{0}$ be Cohen forcing for adding $\kappa_{0}$ Cohen reals and let $\mathbb{P}_{1}$ be the finite support iteration, of length $\kappa_{1}$ of the partial orders $\mathbb{P}\left(\mathcal{B}_{1}\right)$. Let $\mathbb{P}=\mathbb{P}_{0} * \mathbb{P}_{1}$ and let $G$ be $\mathbb{P}$ generic over $V$

Notice first that, since it is easily verified that each member of any $\mathcal{J}_{\mathcal{B}}$ is meager, it follows that the $\kappa_{0}$-Lusin set added by $\mathbb{P}_{0}$ is also an $\left(\mathcal{J}_{\mathcal{B}_{0}}, \kappa_{0}\right)$ Lusin set. From Corollary 1 it follows that $V[G]$ has a $\left(\mathcal{J}_{\mathcal{B}_{0}}, \kappa_{0}\right)$-Lusin set. Hence $\operatorname{cov}\left(\mathcal{J}_{\mathcal{B}_{0}}\right) \geq \kappa_{0}$. Since the continuum in $V[G]$ is $\kappa_{0}$ it follows that $\operatorname{cov}\left(\mathcal{J}_{\mathcal{B}_{0}}\right)=\kappa_{0}$ in this model. On the other hand, it follows from Lemma 3 that $\operatorname{cov}\left(\mathcal{J}_{\mathcal{B}_{1}}\right) \leq \kappa_{1}$. Observe that the finite support iteration $\mathbb{P}_{1}$ adds Cohen reals over each intermediate model. Moreover, as it has already been observed 
that each member of any $\mathcal{J}_{\mathcal{B}}$ is meager, it follows no family of size less than $\kappa_{1}$ elements of $\mathcal{J}_{\mathcal{B}_{1}}$ can cover all the reals. Hence $\operatorname{cov}\left(\mathcal{J}_{\mathcal{B}_{1}}\right)=\kappa_{1}$ in this model.

Definition 5. Define $\mathcal{B}_{n}^{i}$ to consist of all sets

$$
B(a, k)=(\omega \times a) \cup(k \times(n \backslash a))
$$

where $k \in \omega$ and $a \in[n]^{i}$.

It is easy to check that each $\mathcal{B}_{n}^{i}$ is a cover on $\omega \times n$ if $i<n$.

Lemma 4. If $i+1 \leq j$, then $\mathcal{B}_{n}^{i} \prec \mathcal{B}_{m}^{j}$.

Proof. Let $A \in\left(\mathcal{B}_{n}^{i}\right)^{+}$and suppose that $H: A \rightarrow{ }^{\omega}(\omega \times m)$. Without loss of generality it may be assumed that $A=\bigcup_{k \in a} A_{k} \times\{k\}$ where $a \in[n]^{i+1}$ and each $A_{k}$ is infinite. Let $D$ be the set of all $k \in a$ such that the range of $H\left\lceil A_{k} \times\{k\}\right.$ is contained in a finite branching tree. By compactness, for each $k \in D$ there is $c_{k} \in{ }^{\omega}(\omega \times m)$ and $B^{k} \in\left[A_{k} \times\{k\}\right]^{\aleph_{0}}$ such that for all $N \in \omega$ the set $\left\{x \in B^{k}: H(x)\left\lceil N \nsubseteq c_{k}\right\}\right.$ is finite.

For $k \in a \backslash D$ there must exist some $t_{k} \in{ }^{\omega}(\omega \times m)$ such that

$$
\left\{z \in \omega \times m:\left(\exists x \in A_{k} \times\{k\}\right)\left(t_{k}^{\wedge} z \subseteq H(x)\right)\right\}
$$

is infinite. It is then possible to choose $J(k) \in m$ and $B^{k} \in\left[A_{k} \times\{k\}\right]^{\aleph_{0}}$ such that

$$
\left\{z \in \omega \times m:\left(\exists x \in B^{k}\right)\left(t_{k}^{\wedge} z \subseteq H(x)\right)\right\} \subseteq \omega \times\{J(k)\}
$$

and, if $\{x, y\} \in\left[B^{k}\right]^{2}$ and $H(x) \supseteq t_{k} \wedge x^{\prime}$ and $H(y) \supseteq t_{k} \wedge y^{\prime}$, then $x^{\prime} \neq y^{\prime}$. Now let $B_{0}=\bigcup_{k \in a \backslash D} B^{k}$ and $t=\left\{t_{k}: k \in a \backslash D\right\}$. For $s \in t$ let

$$
F(s)=\cup\left\{\omega \times\{J(k)\}: t_{k}=s\right\}
$$

and let $B_{1}=\bigcup_{k \in D} B^{k}$ and $C=\left\{c_{k}: k \in D\right\}$. After shrinking $B^{k}$ further to guarantee that if $k_{0}, k_{1} \in a \backslash D, t_{k_{0}}=t_{k_{1}}, J\left(k_{0}\right)=J\left(k_{1}\right)$ and $x \in B^{k_{0}}$, $y \in B^{k_{1}}$, then $H(x)\left(\left|t_{k_{0}}\right|\right) \neq H(y)\left(\left|t_{k_{0}}\right|\right)$, it is routine to check that these choices witness the required instance of $\mathcal{B}_{n}^{i} \prec \mathcal{B}_{m}^{j}$.

Lemma 5. If $j<n \leq m$, then $\operatorname{cov}\left(\mathcal{J}_{\mathcal{B}_{n}^{j}}\right)=\operatorname{cov}\left(\mathcal{J}_{\mathcal{B}_{m}^{j}}\right)$.

Proof. Let $\beta_{m, n}: \omega \times m \rightarrow \omega \times n$ be a bijection which is the identity on $\omega \times(n-1)$. This induces a bijection $\bar{\beta}_{m, n}:{ }^{\omega}(\omega \times m) \rightarrow{ }^{\omega}(\omega \times n)$ which sends members of the ideal $\mathcal{J}_{\mathcal{B}_{m}^{j}}$ to members of the ideal $\mathcal{J}_{\mathcal{B}_{n}^{j}}$. Hence $\operatorname{cov}\left(\mathcal{J}_{\mathcal{B}_{n}^{j}}\right) \leq$ $\operatorname{cov}\left(\mathcal{J}_{\mathcal{B}_{m}^{j}}\right)$. 
To prove the other inequality, proceed by induction on $n \geq j+1$ to show that $\operatorname{cov}\left(\mathcal{J}_{\mathcal{B}_{n}^{j}}\right) \geq \operatorname{cov}\left(\mathcal{J}_{\mathcal{B}_{n+1}^{j}}\right)$. Now, let $\mathcal{T}$ be a family of no more than $\operatorname{cov}\left(\mathcal{J}_{\mathcal{B}_{n}^{j}}\right)$ many $\mathcal{B}_{n}^{j}$-trees such that ${ }^{\omega}(\omega \times n)=\bigcup_{T \in \mathcal{T}} \bar{T}$. It follows that $\bar{\beta}_{n+1, n}^{-1}(\bar{T})$ is the closure of a $\mathcal{B}_{n+1}^{j+1}$-tree. Next, note that if $T$ is a $\mathcal{B}_{n+1}^{j+1}$-tree, then, by identifying the successor nodes of $T$ with the elements of $\omega \times(j+1)$, it is possible to construct a natural bijection $\gamma_{T}$ from $T$ to ${ }^{\omega}(\omega \times(j+1))$ which also induces a bijection $\bar{\gamma}_{T}: \bar{T} \rightarrow{ }^{\omega}(\omega \times(j+1))$. Furthermore, if $A \in \mathcal{J}_{\mathcal{B}_{j+1}^{j}}$, then $\bar{\gamma}_{T}^{-1}(A) \in$ $\mathcal{J}_{\mathcal{B}_{n+1}^{j}}$. By the induction hypothesis it is known that $\operatorname{cov}\left(\mathcal{J}_{\mathcal{B}_{n}^{j}}\right)=\operatorname{cov}\left(\mathcal{J}_{\mathcal{B}_{j+1}^{j}}\right)$ and so it is possible to find a family $\mathcal{S}_{T}$ of no more than $\operatorname{cov}\left(\mathcal{J}_{\mathcal{B}_{n}^{j}}\right)$ many $\mathcal{B}_{j+1}^{j}$-trees such that ${ }^{\omega}(\omega \times(j+1))=\bigcup_{T \in \mathcal{S}} \bar{T}$. Now, for $T \in \mathcal{T}$ and $S \in \mathcal{S}$ let $B(T, S)=\bar{\gamma}_{T}^{-1} \bar{S}$ and note that $B(T, S)$ is contained in the closure of a $\mathcal{B}_{n+1}^{j}$-tree. Hence $\{B(T, S): T \in \mathcal{T}$ and $\mathcal{S} \in \mathcal{S}\}$ witnesses that $\operatorname{cov}\left(\mathcal{J}_{\mathcal{B}_{n}^{j}}\right) \geq$ $\operatorname{cov}\left(\mathcal{J}_{\mathcal{B}_{n+1}^{j}}\right)$.

Corollary 2. If $j<n, i<m$ and $j>i$, then it is consistent that $\operatorname{cov}\left(\mathcal{J}_{\mathcal{B}_{n}^{j}}\right)<$ $\operatorname{cov}\left(\mathcal{J}_{\mathcal{B}_{m}^{i}}\right)$.

Proof. Apply Lemma 4 and Lemma 5 together with Theorem 1.

As a final remark, notice that an immediate consequence of Theorem 1 and Lemma 1 is that, for any pair of uncountable, regular cardinals $\kappa_{0}>\kappa_{1}$, it is consistent that $\operatorname{cov}(\mathcal{M})=\kappa_{0}$ and $\mathfrak{d}_{1}=\kappa_{1}$ or, in other words, the covering number of the meager ideal is $\kappa_{0}$ while the covering number of the $\mathbb{Q}$-meager ideal is $\kappa_{1}$.

\section{References}

[1] A. Miller. Rational perfect set forcing. In ,Axiomatic Set Theory, (D. A. Martin J. Baumgartner and S. Shelah, editors) Volume 31 of Contemporary Mathematics, 143-159, Providence, 1984. American Mathematical Society.

[2] Arnold W. Miller. Descriptive Set Theory and Forcing, Volume 4 of Lecture Notes in Logic, (1995), Springer, Berlin.

[3] J. Steprāns. Decomposing with smooth sets. Trans. Amer. Math. Soc., (1900), 0:0-0.

[4] J. Steprāns. A very discontinuous Borel function. J. Symbolic Logic,(1993) 58:1268-1283. 
